\title{
Placental alkaline phosphatase levels in cerebrospinal fluid can have a decisive role in the differential diagnosis of intracranial germ cell tumors
}

\author{
Yasuo Aihara, MD, PhD, ${ }^{1}$ Sinichiro Watanabe, MD, PhD, ${ }^{2}$ Kosaku Amano, MD, PhD, ${ }^{1}$ \\ Kana Komatsu, MD, ${ }^{1}$ Kentaro Chiba, MD, ${ }^{1}$ Kosuke Imanaka, MD, ${ }^{1}$ Tomokatsu Hori, MD, PhD, ${ }^{1}$ \\ Takashi Ohba, PhD, ${ }^{2}$ Hitoshi Dairoku, PhD, ${ }^{3}$ Yoshikazu Okada, MD, PhD, ${ }^{1}$ Osami Kubo, MD, PhD, ${ }^{1}$ \\ and Takakazu Kawamata, MD, PhD'1
}

\begin{abstract}
${ }^{1}$ Department of Neurosurgery, Tokyo Women's Medical University, Tokyo; ${ }^{2}$ Division of Internal Medicine and Clinical Laboratory Medicine, Minamisenju Hospital, Tokyo; and ${ }^{3}$ Faculty of Human Sciences, University of Tsukuba, Ibaraki, Japan
\end{abstract}

\begin{abstract}
OBJECTIVE Placental alkaline phosphatase (PLAP) in CSF can provide a very high diagnostic value in cases of intracranial germ cell tumors (GCTs), especially in pure germinomas, to the level of not requiring histological confirmation. Unlike other tumor markers, reliable data analysis with respect to the diagnostic value of PLAP serum or CSF levels has not been available until now. This is the first systematic and comprehensive study examining the diagnostic value of CSF PLAP in patients with intracranial GCTs.
\end{abstract}

METHODS From 2004 to 2014, 74 patients (average age 19.6 \pm 10.6 years) with intracranial GCTs were evaluated using PLAP from their CSF and histological samples. Chemiluminescent enzyme immunoassay was utilized to measure CSF PLAP in the following tumor sites: pineal $(n=32)$, pituitary stalk, suprasellar $(n=16)$, basal ganglia $(n=15)$, intraventricular $(n=9)$, and cerebellar $(n=5)$ regions. In addition to classifying GCT cases, all patients underwent tumor biopsy for correlation with tumor marker data.

RESULTS PLAP in combination with other tumor markers resulted in extremely high sensitivity and specificity of the diagnostic value of intracranial GCTs. Intracranial GCT cases were classified into 1) germinomas, both "pure" and syncytiotrophoblastic giant cell types $(n=38) ; 2)$ nongerminomatous GCTs, choriocarcinomas $(n=9)$ and teratomas $(n=$ 4); and 3) nongerminomas, other kinds of tumors $(n=23)$. Consequently, all patients received chemoradiation therapy based on elevation of PLAP and the histopathological results. It was also speculated that the level of PLAP could show the amount of intracranial germ cell components of a GCT. PLAP was $100 \%$ upregulated in all intracranial germinoma cases. The absence of CSF PLAP proved that the tumor was not a germinoma.

CONCLUSIONS The current study is the first systematic and comprehensive examination of the diagnostic value of the tumor marker PLAP in pediatric patients with intracranial GCT. Using the level of PLAP in CSF, we were able to detect the instances of intracranial germinoma with very high reliability, equivalent to a pathological diagnosis.

https://thejns.org/doi/abs/10.3171/2018.3.JNS172520

KEYWORDS intracranial germ cell tumors; tumor marker; placental alkaline phosphatase; oncology

I NTRACRANIAL germinoma is the most common pediatric brain tumor. ${ }^{15}$ Whereas extragonadal germ cell tumors (GCTs) typically arise in a midline location, the most common sites of intracranial GCTs are the suprasellar, pineal, and basal ganglia regions of the brain..$^{23,26}$ Approximately two-thirds of intracranial GCTs are germi- nomas. ${ }^{14}$ Also belonging to the class of GCTs are embryonal cell carcinomas, yolk sac tumors, teratomas (mature and immature), and choriocarcinomas. ${ }^{14,15,26}$ Germinoma is associated with pituitary dysfunction (diabetes insipidus), visual acuity loss with suprasellar location, and obstructive hydrocephalus with pineal location. ${ }^{14,15,26}$

ABBREVIATIONS AFP $=\alpha$-fetoprotein; $\mathrm{CBDCA}=$ carboplatin; $\mathrm{CDDP}=$ cisplatin; $\mathrm{CEA}=$ carcinoembryonic antigen; $\mathrm{CLEIA}=$ chemiluminescent enzyme immunoassay; $\mathrm{hCG}$ = human chorionic gonadotropin; $\mathrm{hCG}-\beta=\mathrm{hCG} \beta$-subunit; $\mathrm{GCT}$ = germ cell tumor; IgG = immunoglobulin G; PLAP = placental alkaline phosphatase; $\mathrm{STGC}=$ syncytiotrophoblastic giant cell; VP-16 $=$ etoposide.

SUBMITTED October 6, 2017. ACCEPTED March 6, 2018.

INCLUDE WHEN CITING Published online September 28, 2018; DOI: 10.3171/2018.3.JNS172520. 
TABLE 1. Clinical cases: pathological diagnosis and correlation of PLAP value

\begin{tabular}{|c|c|c|c|c|c|}
\hline \multirow{2}{*}{$\begin{array}{l}\text { Pathology } \\
\text { Diagnosis }\end{array}$} & \multirow{2}{*}{$\begin{array}{c}\text { No. of Cases } \\
\quad(n=74)\end{array}$} & \multicolumn{2}{|c|}{ PLAP (pg/ml) } & \multicolumn{2}{|c|}{$\mathrm{hCG}-\beta$ (mlU/ml) } \\
\hline & & Mean & SD & Mean & SD \\
\hline $\begin{array}{l}\text { Germinoma (pure } \\
\quad \& \text { STGC) }\end{array}$ & 38 & 2656.4 & 4692.3 & 4.3 & 7.3 \\
\hline Nongerminoma & 23 & 2.0 & 2.5 & 0.3 & 0.2 \\
\hline $\begin{array}{l}\text { Nongerminoma- } \\
\text { tous GCT (cho- } \\
\text { riocarcinoma) }\end{array}$ & 9 & 3094.7 & 3596.9 & 1610.3 & 2131.8 \\
\hline Teratoma & 4 & 7.6 & 13.0 & 0.4 & 0.3 \\
\hline
\end{tabular}

The diagnosis of intracranial tumors is fundamentally based on tumor histology and cytology obtained by an invasive procedure. Small tumor samples do not reflect malignancy correctly.,11 Tumor markers (such as $\alpha$-fetoprotein [AFP] or human chorionic gonadotropin [hCG]) are useful diagnostic markers in the absence of tumor biopsy. ${ }^{1,2,6}$

Intracranial GCTs are highly responsive to chemotherapy and radiation therapy, and that makes surgical cytology indispensable. ${ }^{10,13}$ Advances in radiation therapy techniques complemented by chemotherapy have improved outcomes in these patients. ${ }^{7,8,16}$ Before treatment, histological confirmation is recommended and is usually performed by endoscopic or stereotactic methods, or open biopsy. However, occasionally biopsy may be difficult to perform due to the deep-seated location of GCTs, and/or the diagnosis might remain unconfirmed because of insufficient samples for proper histopathology. Additionally, biopsy procedures are invasive, with the associated risks of bleeding and infection. ${ }^{4,18,22,29}$

Placental alkaline phosphatase (PLAP) exists in trophoblast cells of normal human mature placenta, seminomas of testis, and ovarian carcinomas. ${ }^{3}$ It is well known that PLAP immunohistochemical identification is useful

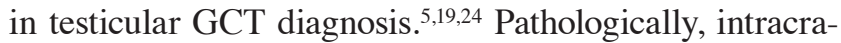
nial GCTs are similar to testicular and other extragonadal GCTs. ${ }^{20,21,27}$ Shinoda et al. first reported that PLAP activity was increased in serum and/or CSF of patients with primary intracranial germinomas. ${ }^{21}$ Ono et al. also published a study in which 5 of 9 patients with germinomas had high CSF PLAP levels, suggesting that PLAP could be a useful tumor marker for the diagnosis of germinomas. ${ }^{17}$ However, unlike other tumor markers, reliable data analysis has not been performed with respect to the diagnostic value of PLAP serum or CSF levels.

Although PLAP could be measured in serum, CSF levels are a more sensitive and reliable measure of tumor presence. PLAP has already been reported as a highly sensitive and specific chemiluminescent enzyme immunoassay (CLEIA) in CSF for patients with intracranial GCT. ${ }^{28}$ If accurate preoperative diagnosis of intracranial GCTs can be accomplished with high sensitivity by measuring PLAP, radio-/chemotherapy may be started without resorting to biopsy. With the possession of such a highly sensitive method, we also attempted a new intracranial GCT diagnostic approach based on multitumor marker com-
TABLE 2. Tumor locations

\begin{tabular}{lc}
\hline \multicolumn{1}{c}{ Location } & No. of Cases \\
\hline Pineal & 28 \\
\hline Parasellar & 13 \\
\hline Parasellar, pineal & 9 \\
\hline Thalamus & 9 \\
\hline Basal ganglia & 3 \\
\hline Third ventricle & 3 \\
\hline Parasellar, basal ganglia & 2 \\
\hline Cerebellum & 1 \\
\hline Cerebellum, basal ganglia & 1 \\
\hline Multiple intraventricular & 1 \\
\hline Parasellar, pineal, ventricle & 1 \\
\hline Periventricular, peribrainstem & 1 \\
\hline Pineal, apex of pyramidal bone & 1 \\
\hline Tectum & 1 \\
\hline Total & 74 \\
\hline
\end{tabular}

prehensive diagnosis including PLAP using retrospective analysis of CSF marker levels for patients with intracranial GCT.

\section{Methods \\ CSF Samples}

PLAP CSF levels in intracranial GCTs were retrospectively analyzed using archival CSF samples, which were obtained preoperatively via lumbar taps or aspirated during surgery and stored at $-80^{\circ} \mathrm{C}$ at the Department of Neurosurgery, Tokyo Women's Medical University Hospital. Between 2004 and 2014, 74 patients were investigated and classified (Table 1). The average patient age was 19.6 \pm 10.6 years with a male to female ratio of 59:15. Tumor locations were predominantly along the midline (Table 2). Blood-contaminated CSF samples and patients who had already received chemotherapy/radiation therapy at previous hospitals were excluded to avoid technical errors.

Final diagnoses of the patients were established by histological and immunohistochemical studies or by CSF cytology analysis. For the purpose of the study, these diagnoses were distributed into groups according to the WHO Classification of Tumors of the CNS (2016). In addition, the PLAP concentrations were determined before and after conventional therapy in all patients with germinoma who achieved clinical complete remission with radio-/ chemotherapy. ${ }^{28}$ This study was approved by the Human Investigation Committee of the Tokyo Women's Medical University Hospital and performed in accordance with the Declaration of Helsinki.

\section{CLEIA Procedure for Measuring PLAP}

Monoclonal antibodies against purified human PLAP were prepared according to conventional procedures. ${ }^{12}$ Four monoclonal antibodies were obtained and shown to react with both Regan- and Nagao-type alkaline phosphatases to a similar degree. MoAb24, one of the four anti- 

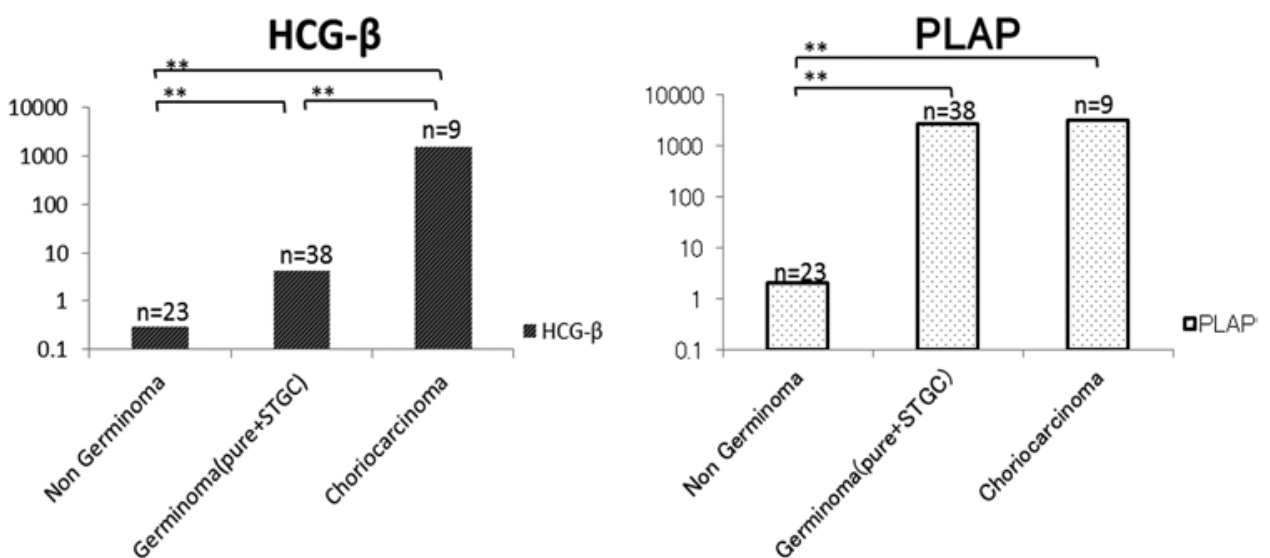

FIG. 1. Bar graphs showing the differences between each tumor marker group. Differences were significant for both variables and every dependent variable: PLAP level, $F(3,70)=89.0, p<0.001$, and hCG- $\beta$ level, $F(3,70)=70.5, p<0.001 .{ }^{* *} p<0.001$.

human PLAP monoclonal antibodies, was used for this CLEIA. A monoclonal antibody against mouse immunoglobulin $\mathrm{G}$ ( $\mathrm{IgG}$ ) was obtained from Fujirebio Inc. Ferrite particles $(0.3 \mu \mathrm{m}$ in diameter; Nippon Print Co.) were coated with the anti-mouse IgG antibody. Afterward, the ferrite particles were coated with bovine serum albumin (Oriental Yeast Co. Ltd.). AMPPD (3-[2'-spiroadamantyl]4-methoxy-4-[3"-phosphoryloxy]-phenyl-1,2-dioxetane) was used as the chemiluminescent substrate for the alkaline phosphatase. Human PLAP (Calzyme Laboratories Inc.) was used as a standard and diluted in Tris-buffered saline to concentrations of 5, 10,25, 50, 100,250,500, and $1000 \mathrm{pg} / \mathrm{ml}$, respectively.

The $95 \%$ confidence interval was determined by measuring 3 PLAP standards $(0,5$, or $10 \mathrm{pg} / \mathrm{ml}) 20$ times each. Intra- and interassay variations were determined by measuring different PLAP standards 10 times each. Crossreactivity of the anti-human PLAP monoclonal antibody (MoAb24) was tested against human liver, bone, and intestinal alkaline phosphatase (Calzyme Laboratories).

\section{Results}

We measured the tumor marker levels of 78 patients and initially identified embryonal carcinomas and yolk sac tumors by their increased levels of AFP and carcinoembryonic antigen (CEA). Four tumors matched their high AFP levels with yolk sac tumor histological diagnosis; however, we did not have an embryonal carcinoma in our series. Furthermore, dependent variable and multivariate ANOVA was undertaken with an independent variable for PLAP and hCG $\beta$-subunit (hCG- $\beta$ ) levels, according to pathological diagnosis (Figs. 1 and 2).

\section{PLAP in CSF as Differential Diagnosis}

PLAP levels in CSF were measured in a total of 74 patients with histopathological diagnoses: 1) germinomas, both "pure" and syncytiotrophoblastic giant cell (STGC) types $(\mathrm{n}=38) ; 2)$ nongerminomatous GCTs, choriocarcinomas $(\mathrm{n}=9)$ and teratomas $(\mathrm{n}=4)$; and 3$)$ non-GCTs, other kinds of tumors $(n=23)$. The correlation of PLAP to histopathological diagnosis showed extremely high sen- sitivity and specificity without false-positive or -negative cases. Regarding the results of the dependent variable and the multivariate analysis, the difference between groups was significant $(\mathrm{p}<0.001)$. The difference between groups was significant for both variables when we examined every dependent variable: PLAP level, $\mathrm{p}<0.001$, and hCG- $\beta$ level, $\mathrm{p}<0.001$ (Fig. 1). The results of multiple comparisons (using the Bonferroni method) for germinomas and choriocarcinomas showed significantly higher PLAP and hCG- $\beta$ levels compared with nongerminomas $(p<0.01)$. In addition, germinomas had significantly lower hCG- $\beta$ levels compared with the choriocarcinoma group ( $\mathrm{p}<$ 0.01 ; Fig. 2).

Thus, an hCG- $\beta>50 \mathrm{mIU} / \mathrm{ml}$ was clearly indicative of the presence of choriocarcinoma, and a moderate increase up to $50 \mathrm{mIU} / \mathrm{ml}$ suggested a germinoma with STGC, the last group also demonstrating significant values of PLAP $>100 \mathrm{pg} / \mathrm{ml}$. Pure germinomas and teratomas were negative for hCG- $\beta$, and the levels of PLAP depended on the presence of germinomatous tissue, as either pure germinomas or immature teratomas. Mature teratomas were negative for both PLAP and hCG- $\beta$ (Fig. 3A).

\section{Discussion}

Within pediatric brain tumors, intracranial GCTs can be classified according to their histological picture of WHO classification: germinomas (both pure and those containing STGC), teratomas (mature and immature), teratomas with malignant transformation, yolk sac tumors, embryonal carcinomas, and choriocarcinomas. The last three types of tumors usually contain elements of mixed GCTs. Intracranial GCTs have immunohistochemical features similar to those of gonadal GCTs. These features involve hCG-which is present in embryonal cell carcinomas, choriocarcinomas, and their mixed forms-and AFP (nongerminomatous GCT), which is also present in embryonal cell carcinomas, as well as in yolk sac tumors. These tumor products can also be detected in serum or CSF and are complements to the diagnosis of a GCT and predictors of tumor histology., ${ }^{1,6,13}$ Pure germinomas do not contain hCG and AFP at all. We postulated that PLAP in CSF alone could provide an accurate diagnosis 


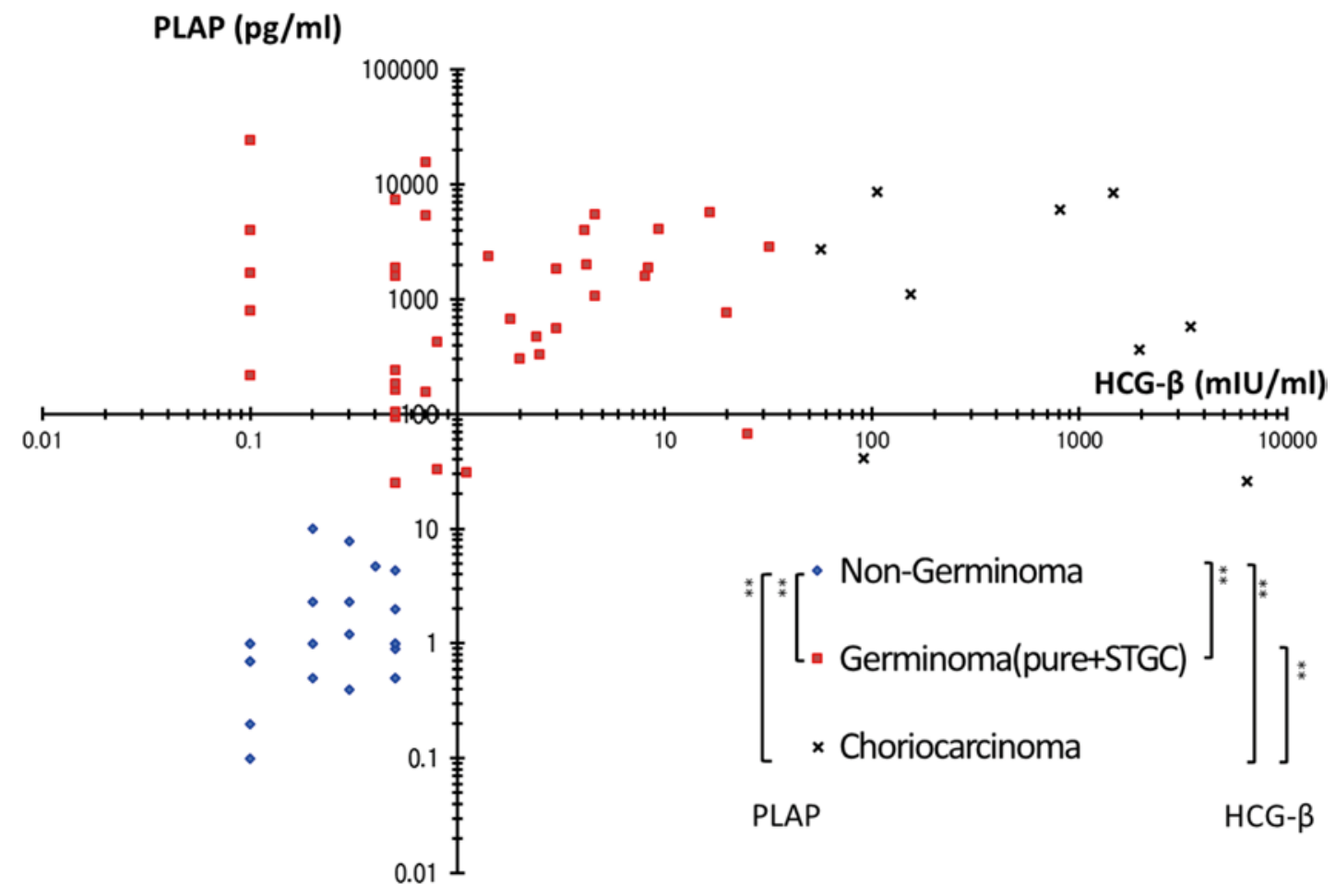

FIG. 2. Scatterplot showing that results of multiple comparisons (using the Bonferroni method) for germinomas and choriocarcinomas were significantly higher for the PLAP and hCG- $\beta$ levels together compared with those for nongerminomas $(p<0.01)$. In addition, compared with the choriocarcinoma group, germinomas only had significantly lower hCG- $\beta$ levels $(p<0.01)$.

in pure germinomas, and histological confirmation might not be required in the future to establish the diagnosis. In this report, we present 74 patients with intracranial GCTs, mainly in the suprasellar region and pineal area, with or without obstructive hydrocephalus. All patient diagnoses were confirmed by histological examination after obtaining open and/or neuroendoscopic biopsies from the tumor.

Initial clinical diagnosis in every case was established on the basis of clinical data and the findings on MRI relevant to a tumor, later verified by histology. The diagnosis of the tumors in this series could be confirmed on the basis of the typical clinical and imaging presentation complemented by tumor marker expression, mainly PLAP, with extremely high sensitivity and specificity (Fig. 2). We also transferred these results in the existing intracranial GCT classification based on pathological diagnosis (Fig. 3A). In all pure germinoma and germinoma with STGC cases, tumors disappeared after chemotherapy and radiation treatment. No recurrence developed during a followup of more than 5 years.

The presenting signs and symptoms of intracranial germinomas depend on the location of the tumor. Intracranial germinomas favor midline locations such as the suprasellar and pineal regions. Suprasellar lesions commonly present with visual field defects due to compression of the optic chiasm, diabetes insipidus, and other signs of hypothalamic-pituitary dysfunction, and those in the pineal gland with neurological dysfunction caused by intracranial hypertension due to direct invasion or obstruction of the CSF outflow, including headaches, visual abnormalities, papilledema, ataxia, Parinaud syndrome, tremor, and altered pupillary reflexes. ${ }^{2,9}$ These case histories raise the following question: can a diagnosis of germinoma be made on the basis of an elevation of PLAP CSF levels only, without histological verification? The differential diagnosis of suprasellar tumors comprises craniopharyngiomas, optic gliomas, sarcoidosis, GCTs, histiocytosis X, hemochromatosis, and lymphocytic hypophysitis. From our study, it becomes evident that a PLAP investigation of the sample of CSF obtained during hydrocephalus treatment can provide early indications of the histological presence of germinomatous cell populations and the needed treatment protocol. According to the differential diagnosis of suprasellar and pineal region tumors, the only possible diagnosis in cases of multiple midline tumors is a GCT. ${ }^{16}$

Therefore, in the case of an intracranial tumor with the above-mentioned clinical and imaging characteristics, with normal values of the tumor markers hCG and AFP in serum and elevated PLAP in CSF, the only possible diagnosis is a germinoma. We consider that under such circumstances no histological analysis is required to confirm the diagnosis. This suggestion is consistent with published reports of multiple midline tumors in which biopsy revealed the histological picture of germinoma in all cases with normal tumor markers, excluding PLAP. ${ }^{11,14}$ In contrast, if each of the hCGs or germinomas with STGC, AFP, and CEA is elevated in serum or CSF, these kinds of tumors could be diagnosed as choriocarcinoma, yolk sac tumor, and embryonal cell carcinoma, respectively. In such a situation a biopsy is not needed either. In the case of elevation of hCG, AFP, and CEA combined with the PLAP value, a differential diagnosis could be performed, 

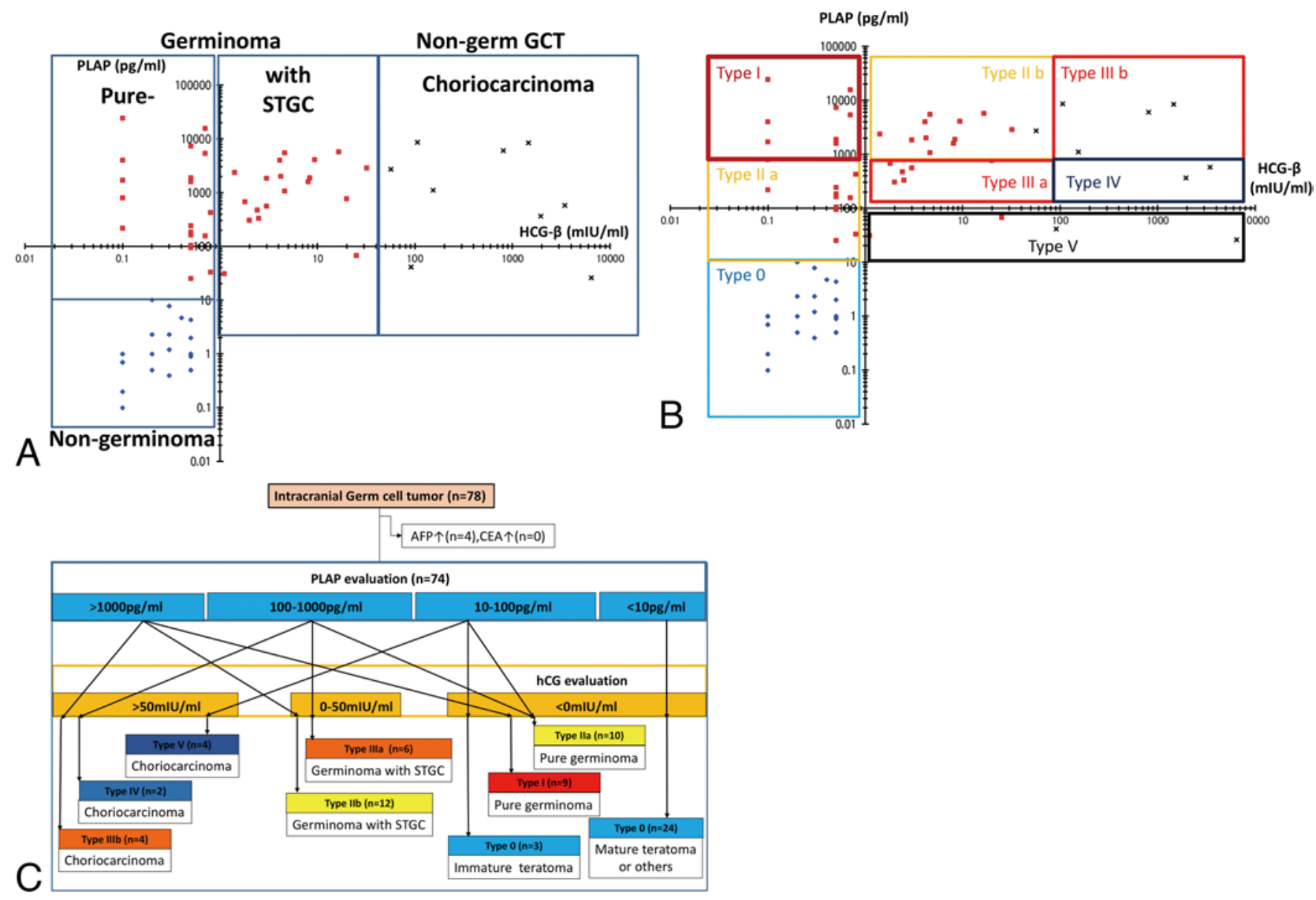

FIG. 3. A: Scatterplot that classified the value of the PLAP/hCG- $\beta$ marker combination according to the current intracranial GCT grade classification. B: Scatterplot showing the new intracranial GCT classification (types $0-V$ ) based only on PLAP and hCG- $\beta$ value combination. C: Diagnostic flowchart indicating (with lines) the type of tumor according to a combination of value ranges of hCG and PLAP. As can be observed, some of the histological entities demonstrated more than one type of tumor marker characteristic and are presented as separate subtypes. Their clinical significance will be investigated later.

as summarized in Table 3. In this way, a new, more detailed classification of intracranial GCTs can be proposed based on 2D representation of tumor marker values, as the reliability of PLAP as a marker was demonstrated comparing these results with pathology findings. This new intracranial GCT classification, including types $0-\mathrm{V}$ (Fig. $3 \mathrm{~B}$ ), is based only on PLAP and hCG- $\beta$ (AFP) value combination and has the potential to be used for the selection of a treatment protocol.

This differential diagnostic approach is particularly important for the most significant decision on the treatment protocol, that is, to continue with radio- and chemotherapy, or consider surgical removal as the next option, when final histological confirmation will be obtained.

In an attempt at further differentiation, a new classification can be defined as follows: 1) type $0=$ negative $\operatorname{PLAP}(<10 \mathrm{pg} / \mathrm{ml})$ value and normal hCG- $\beta$ range, which is the nongerminoma group; 2 ) type I = high PLAP value and normal hCG- $\beta$ range, which is the high-degree, pure germinoma group; 3 ) type IIa = mild-moderate elevation of PLAP (10-1000 pg/ml) value and normal hCG- $\beta$ range, which is the mild- to moderate-degree, pure germinoma group; 4) type IIb = high PLAP (>1000 pg/ml) value and moderately elevated hCG- $\beta(<100 \mathrm{mIU} / \mathrm{ml})$, which indicates the mixed germinoma with a high proportion of germinomatous components group; 5) type IIIa = mildmoderate elevation of PLAP (10-1000 pg/ml) value and

TABLE 3. New differential diagnosis of intracranial GCTs in relation to existing tumor markers and PLAP

\begin{tabular}{ccccc}
\hline & PLAP & hCG- $\beta$ & AFP & CEA \\
\hline Germinoma & & & & \\
\hline Pure & +++ & - & - & - \\
\hline w/ STGC & ++ & + & - & - \\
\hline Choriocarcinoma & + & ++ & - & - \\
\hline Yolk sac tumor & + & - & ++ & - \\
\hline Embryonal carcinoma & + & - & - & + \\
\hline Teratoma & & & & - \\
\hline Mature & - & - & - & - \\
\hline Immature & $+/-$ & - & + & - \\
\hline
\end{tabular}

Symbols indicate the degree of elevation of the marker level, from lowest (-) to highest (+++). 
moderately elevated hCG- $\beta(<100 \mathrm{mIU} / \mathrm{ml})$, which indicates the mixed germinoma with a low amount of germinomatous components group; 6$)$ type IIIb = high PLAP (> $1000 \mathrm{pg} / \mathrm{ml})$ value and high hCG- $\beta(>100 \mathrm{mIU} / \mathrm{ml})$, which indicates the choriocarcinoma with a high amount of germinomatous components group; 7) type IV = mild-moderate elevation of PLAP (10-1000 pg/ml) value and high hCG- $\beta$ (> $100 \mathrm{mIU} / \mathrm{ml}$ ), which is the choriocarcinoma with a low amount of germinomatous components group; and 8) type $\mathrm{V}=$ mild elevation of PLAP (10-100 pg/ml) value and moderate-high hCG- $\beta$ (>0 mIU/ml), which is the clear choriocarcinoma with a very low amount of germinomatous components group. The diagnostic algorithm based on tumor markers could be summarized as shown in Fig. 3C.

Hopefully, a new protocol of conventional chemo-/radiotherapy could be designed based on the new classification for each group and further clinical correlations for these new types of tumors will be performed.

\section{Illustrative Cases Based on the New Classification Case 1 (Type I)}

A 15-year-old boy with a history of bilateral visual impairment followed by progressive headaches underwent MRI, which showed hydrocephalus caused by large pineal lesions. No AFP or hCG could be detected in the CSF except the high elevation $(>1000 \mathrm{pg} / \mathrm{ml})$ of PLAP of 2230 $\mathrm{pg} / \mathrm{ml}$. The patient received chemotherapy (carboplatin [CBDCA] and etoposide [VP-16]) as first-line treatment. MRI of the brain at 2 and 3 weeks after the first course of chemotherapy revealed that the abnormality at the pineal region had disappeared (Fig. 4A). This shows that the tumor itself is of high chemosensitivity because the purity of GCT is high in type I.

\section{Case 2 (Type IIIb)}

A 14-year-old girl presented with a history of progressive headaches with nausea and vomiting. At the same time, she complained of polyuria caused by diabetes insipidus. MRI showed hydrocephalus caused by a huge mass occupying the suprasellar region and third ventricle. The hCG $(-\beta)$ and PLAP levels in the CSF on the day of admission were $2280(2500) \mathrm{mIU} / \mathrm{ml}$ and $8414 \mathrm{pg} / \mathrm{ml} \mathrm{(>} 1000$ $\mathrm{pg} / \mathrm{ml}$ ), respectively. The patient received chemotherapy (CBDCA and VP-16) as first-line treatment. MRI of the brain at 2 and 3 weeks after chemotherapy revealed that the huge mass lesion had disappeared and hydrocephalus improved without ventriculoperitoneal shunt intervention (Fig. 4B). This demonstrated that the tumor itself was highly responsive due to chemosensitivity, even though the pathology findings showed choriocarcinoma components, because the amount of pure GCT was very high as defined in tumors of type IIIb.

\section{Case 3 (Type V)}

A 13-year-old boy was admitted with a history of progressive right hemimotor weakness. MRI showed an enhanced mass in the left basal ganglia. The hCG(- $\beta)$ and PLAP levels in the CSF on the day of admission were 86 (91) $\mathrm{mIU} / \mathrm{ml}$ and $41 \mathrm{pg} / \mathrm{ml}(<100 \mathrm{pg} / \mathrm{ml})$, respectively.
The patient received chemotherapy (cyclophosphamide, cisplatin [CDDP], and VP-16) as the first line of treatment. MRI of the brain at 2 and 4 weeks after the first course of chemotherapy revealed poor mass reduction of the tumor compared with initial MRI (Fig. 4C). For a low presence of germ cells for a tumor of type V, in which low PLAP values exist, this shows the tumor had poor chemosensitivity despite the pathological finding of STGC components.

\section{Case 4 (Type V)}

An 18-year-old boy presented with a history of progressive headaches with nausea and vomiting. MRI showed hydrocephalus caused by a pineal lesion. On the day of admission, the hCG(- $\beta$ ) and PLAP levels were 6421 (6246) $\mathrm{mIU} / \mathrm{ml}$ and $15 \mathrm{pg} / \mathrm{ml}(<100 \mathrm{pg} / \mathrm{ml})$, respectively. The patient received chemotherapy (cyclophosphamide, CDDP, and VP-16) as a first line of treatment. MRI of the brain at 2 and 4 weeks after the first course of chemotherapy revealed poor mass reduction of the tumor compared with the initial MRI (Fig. 4D). This tumor also showed poor chemosensitivity and the pathology findings showed choriocarcinoma components with a high MIB-1 index. This is one of the most highly malignant GCT groups (type V), which contains massive choriocarcinoma portions with a very low amount of pure GCT components.

As of 2004, our department has used this original classification based on CSF PLAP levels in conjunction with the other routine tumor markers (hCG- $\beta$, AFP, and CEA) for the management of intracranial GCTs. It is important to mention that the biological behavior of germinomas with STGC has not been well established. The limited information in the literature suggests that intracranial germinomas with STGC producing hCG are associated with a higher recurrence rate compared with pure germinomas without such production. Germinomas with STGC and serum hCG levels higher than $15 \mathrm{mIU} / \mathrm{ml}$ have a high recurrence rate, and more aggressive treatment is indicated for these patients. ${ }^{25}$ It is difficult to determine whether the poorer prognosis for germinomas with STGC is inevitable, and the cutoff value of hCG for determining such prognosis is still unknown. Regardless of the characteristics of germinoma with STGC, in future practice PLAP potentially could become the single significant tumor marker for the diagnosis of intracranial germinomas without histological verification.

In this report, the number of immature and mature teratoma cases $(n=4)$ was very small compared with the rest of the tumor types. Notably, their PLAP values were 7.6 $\mathrm{pg} / \mathrm{ml}$ on average, indicating teratomas. Immature teratomas could include some PLAP-producing cell populations. Based on this evidence, it could be speculated that the PLAP value itself reflected the presence of germ cell populations and, consequently, sensitivity to chemoradiotherapy. It can be inferred that the setting of cutoff values of PLAP can play an important role in the diagnostic workup. PLAP values can also be useful for choosing the endpoint of clinical treatment, especially chemoradiotherapy, because differentiation between residual tumor and the scar of tumor tissue can be difficult on MRI and methionine PET imaging. 


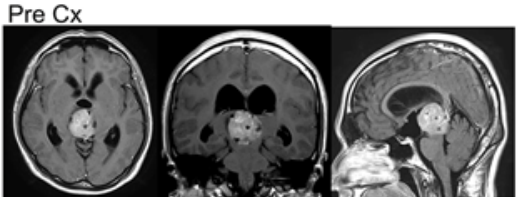

Post CX 2week

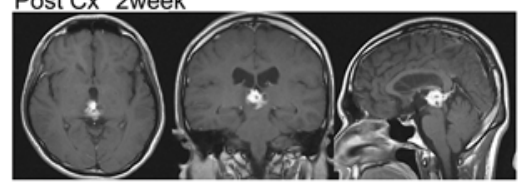

PLAP: 2230pg/ml

hCG $(-\beta)$ : normal value

\section{Type I}

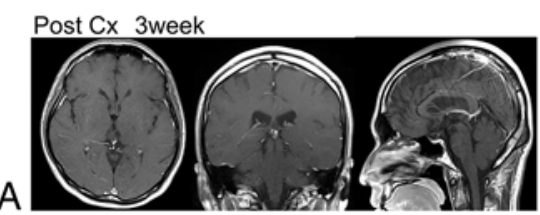

Cx:Chemotherapy (CBDCA, VP16)

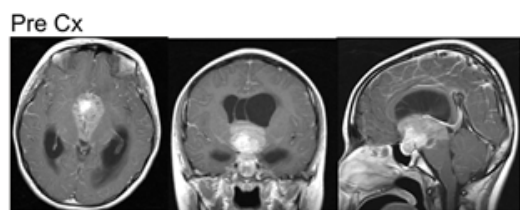

Post Cx 2week

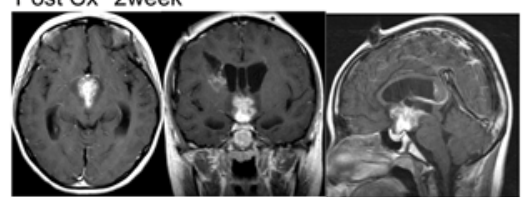

PLAP: $8414 \mathrm{pg} / \mathrm{ml}$

hCG-B: $2500 \mathrm{mIU} / \mathrm{ml}$

hCG: $2280 \mathrm{mlU} / \mathrm{ml}$

Type III b

Cx:Chemotherapy (CBDCA, VP16)

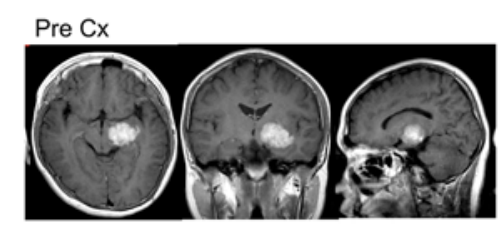

Post Cx 2week

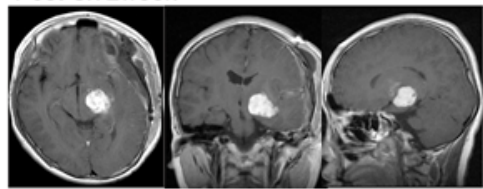

Post Cx 4week

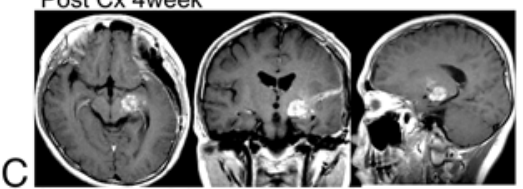

PLAP: $41 \mathrm{pg} / \mathrm{ml}$

hCG- $\beta$ : $91 \mathrm{mlU} / \mathrm{ml}$ hCG: $86 \mathrm{mlU} / \mathrm{ml}$

\section{Type V}

Cx:Chemotherapy
(Cyclophosphamide, (Cyclophospham
CDDP, VP16)
B
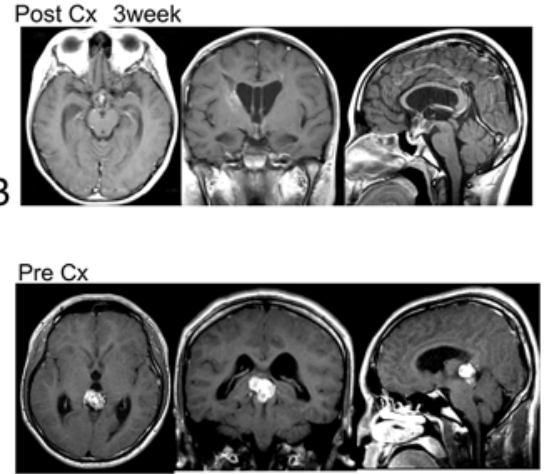

Post Cx 2week
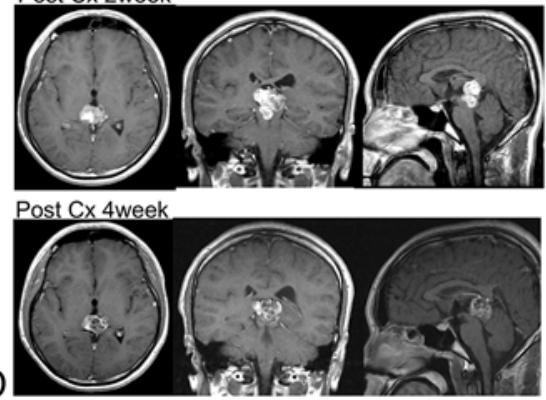

PLAP: $15 \mathrm{pg} / \mathrm{ml}$

hCG- $\beta$ : $6246 \mathrm{mlU} / \mathrm{ml}$ hCG: $6421 \mathrm{mlU} / \mathrm{ml}$

\section{Type V}

Cx:Chemotherapy (Cyclophosphamide,
CDDP, VP16)

FIG. 4. A: Case 1 (type I). T1-weighted Gd-enhanced MRI demonstrates acute hydrocephalus and a huge mass in the pineal region. Just 2-3 weeks after the first course of chemotherapy (CBDCA/VP-16), new MRI demonstrates the complete remission of the pineal region tumor. B: Case 2 (type IIlb). T1-weighted Gd-enhanced MRI demonstrates acute hydrocephalus and a huge mass in the suprasellar region and third ventricle. Just 2-3 weeks after the first course of chemotherapy (CBDCA/VP-16), the massive tumor disappeared rapidly and hydrocephalus improved without ventriculoperitoneal shunt placement. C: Case 3 (type V). T1-weighted Gd-enhanced MRI demonstrates a mass in the left basal ganglia. MRI of the brain at 2 and 4 weeks after the first course of chemotherapy revealed poor mass reduction of the tumor compared with the initial MRI. D: Case 4 (type V). T1-weighted Gd-enhanced MRI demonstrates acute hydrocephalus and tumor mass in the pineal region. MRI of the brain at 2 and 4 weeks after the first course of chemotherapy revealed poor mass reduction of the tumor compared with the initial MRI. Figure is available in color online only.

\section{Conclusions}

As the first systematic and comprehensive examination of the diagnostic value of the tumor marker PLAP in CSF in pediatric patients with intracranial GCT, this study demonstrated that PLAP in CSF shows promise as a novel tumor marker. CSF PLAP possesses a high diagnostic value in the approach to intracranial germinoma cases, and accurate diagnostic power equivalent to pathological diagnosis. This can permit a future in which the therapeutic strategy for intracranial GCT case management bypasses invasive techniques that could be based on the establishment of diagnostic criteria of new CSF tumor markers, where PLAP could play an essential role. We believe that such a diagnostic approach could reduce the number of invasive surgical procedures aiming at the establishment of histological diagnosis.

\section{Acknowledgments}

We thank Dr. Kostadin Karagiozov and David Huang for their guidance and gratefully acknowledge the radiological technologist Dr. Komori and Mr. Sakayori (pathological technologists) and staff of the Department of Neurosurgery, Tokyo Women's Medical University, for their assistance in preparing this paper. This study was supported by research funds from the Department of Neurosurgery, Tokyo Women's Medical University.

\section{References}

1. Aihara Y, Kashiwase S, Miki N, Kimura T, Okada Y: [Pitfall 
of the blood HCG value in the suprasellar region germinoma.] No Shinkei Geka 41:65-69, 2013 (Jpn)

2. Allen JC, Nisselbaum J, Epstein F, Rosen G, Schwartz MK: Alphafetoprotein and human chorionic gonadotropin determination in cerebrospinal fluid. An aid to the diagnosis and management of intracranial germ-cell tumors. J Neurosurg 51:368-374, 1979

3. Beckstead JH: Alkaline phosphatase histochemistry in human germ cell neoplasms. Am J Surg Pathol 7:341-349, 1983

4. Choi UK, Cha SH, Song GS, Choi CH, Lee SW, Lim YT, et al: Recurrent intracranial germinoma along the endoscopic ventriculostomy tract. Case report. J Neurosurg 107 (1 Suppl):62-65, 2007

5. Friedman NB: Germinoma of the pineal; its identity with germinoma (seminoma) of the testis. Cancer Res 7:363-368, 1947

6. Fujimaki T, Mishima K, Asai A, Tabuchi K, Kobayashi M, Suzuki I, et al: Levels of beta-human chorionic gonadotropin in cerebrospinal fluid of patients with malignant germ cell tumor can be used to detect early recurrence and monitor the response to treatment. Jpn J Clin Oncol 30:291-294, 2000

7. Haas-Kogan DA, Missett BT, Wara WM, Donaldson SS, Lamborn KR, Prados MD, et al: Radiation therapy for intracranial germ cell tumors. Int J Radiat Oncol Biol Phys 56:511-518, 2003

8. Hardenbergh PH, Golden J, Billet A, Scott RM, Shrieve DC, Silver B, et al: Intracranial germinoma: the case for lower dose radiation therapy. Int J Radiat Oncol Biol Phys 39:419-426, 1997

9. Hirato J, Nakazato Y: Pathology of pineal region tumors. J Neurooncol 54:239-249, 2001

10. Huh SJ, Shin KH, Kim IH, Ahn YC, Ha SW, Park CI: Radiotherapy of intracranial germinomas. Radiother Oncol 38:19-23, 1996

11. Jennings MT, Gelman R, Hochberg F: Intracranial germcell tumors: natural history and pathogenesis. J Neurosurg 63:155-167, 1985

12. Köhler G, Milstein C: Continuous cultures of fused cells secreting antibody of predefined specificity. Nature 256:495497, 1975

13. Lafay-Cousin L, Millar BA, Mabbott D, Spiegler B, Drake J, Bartels U, et al: Limited-field radiation for bifocal germinoma. Int J Radiat Oncol Biol Phys 65:486-492, 2006

14. Matsutani M, Sano K, Takakura K, Fujimaki T, Nakamura $\mathrm{O}$, Funata N, et al: Primary intracranial germ cell tumors: a clinical analysis of 153 histologically verified cases. J Neurosurg 86:446-455, 1997

15. Nomura K: Epidemiology of germ cell tumors in Asia of pineal region tumor. J Neurooncol 54:211-217, 2001

16. Ogawa K, Shikama N, Toita T, Nakamura K, Uno T, Onishi $\mathrm{H}$, et al: Long-term results of radiotherapy for intracranial germinoma: a multi-institutional retrospective review of 126 patients. Int J Radiat Oncol Biol Phys 58:705-713, 2004

17. Ono N, Naganuma H, Inoue HK, Tamura M: Cerebrospinal fluid placental alkaline phosphatase in the intracranial germinomas: results of enzyme antigen immunoassay. Neurol Med Chir (Tokyo) 31:563-567, 1991

18. Oppido PA, Fiorindi A, Benvenuti L, Cattani F, Cipri S, Gangemi M, et al: Neuroendoscopic biopsy of ventricular tumors: a multicentric experience. Neurosurg Focus 30(4):E2, 2011

19. Paiva J, Damjanov I, Lange PH, Harris H: Immunohisto- chemical localization of placental-like alkaline phosphatase in testis and germ-cell tumors using monoclonal antibodies. Am J Pathol 111:156-165, 1983

20. Russell DS: "Ectopic pinealoma": its kinship to atypical teratoma of the pineal gland; report of a case. J Pathol Bacteriol 68:125-129, 1954

21. Shinoda J, Yamada H, Sakai N, Ando T, Hirata T, Miwa Y: Placental alkaline phosphatase as a tumor marker for primary intracranial germinoma. J Neurosurg 68:710-720, 1988

22. Shono T, Natori Y, Morioka T, Torisu R, Mizoguchi M, Nagata S, et al: Results of a long-term follow-up after neuroendoscopic biopsy procedure and third ventriculostomy in patients with intracranial germinomas. J Neurosurg 107 (3 Suppl):193-198, 2007

23. Sugiyama K, Uozumi T, Kiya K, Mukada K, Arita K, Kurisu $\mathrm{K}$, et al: Intracranial germ-cell tumor with synchronous lesions in the pineal and suprasellar regions: report of six cases and review of the literature. Surg Neurol 38:114-120, 1992

24. Uchida T, Shimoda T, Miyata H, Shikata T, Iino S, Suzuki $\mathrm{H}$, et al: Immunoperoxidase study of alkaline phosphatase in testicular tumor. Cancer 48:1455-1462, 1981

25. Utsuki S, Kawano N, Oka H, Tanaka T, Suwa T, Fujii K: Cerebral germinoma with syncytiotrophoblastic giant cells: feasibility of predicting prognosis using the serum hCG level. Acta Neurochir (Wien) 141:975-978, 1999

26. van Battum P, Huijberts MS, Heijckmann AC, Wilmink JT, Nieuwenhuijzen Kruseman AC: Intracranial multiple midline germinomas: is histological verification crucial for therapy? Neth J Med 65:386-389, 2007

27. Wahren B, Holmgren PA, Stigbrand T: Placental alkaline phosphatase, alphafetoprotein and carcinoembryonic antigen in testicular tumors. Tissue typing by means of cytologic smears. Int J Cancer 24:749-753, 1979

28. Watanabe S, Aihara Y, Kikuno A, Sato T, Komoda T, Kubo $\mathrm{O}$, et al: A highly sensitive and specific chemiluminescent enzyme immunoassay for placental alkaline phosphatase in the cerebrospinal fluid of patients with intracranial germinomas. Pediatr Neurosurg 48:141-145, 2012

29. Wellons JC III, Reddy AT, Tubbs RS, Abdullatif H, Oakes WJ, Blount JP, et al: Neuroendoscopic findings in patients with intracranial germinomas correlating with diabetes insipidus. J Neurosurg 100 (5 Suppl Pediatrics):430-436, 2004

\section{Disclosures}

The authors report no conflict of interest concerning the materials or methods used in this study or the findings specified in this paper.

\section{Author Contributions}

Conception and design: Aihara, Okada. Acquisition of data: Aihara, Amano, Chiba, Imanaka, Ohba, Kubo. Analysis and interpretation of data: Aihara, Watanabe, Komatsu, Dairoku. Drafting the article: Aihara. Critically revising the article: Aihara. Reviewed submitted version of manuscript: Aihara. Approved the final version of the manuscript on behalf of all authors: Aihara. Statistical analysis: Aihara. Administrative/technical/material support: Hori. Study supervision: Kawamata.

\section{Correspondence}

Yasuo Aihara: Tokyo Women's Medical University, Tokyo, Japan. yaihara@twmu.ac.jp; yz3y-aihr@asahi-net.or.jp. 